\title{
Obesidade e sua Relação com Fatores de Risco para Doenças Cardiovasculares em uma População Nipo-Brasileira
}

artigo original

\author{
Roberta Carvalho de Figueiredo \\ LaÉrCIO Joel Franco \\ RegINA C. GarCIA DE ANDRADE \\ Maria CRISTINA Foss-Freitas \\ Ana EMilia Pace \\ Amaury Lelis Dal FabBro \\ MILTON CÉsAR Foss
}

Faculdade de Medicina da Universidade Federal de Minas Gerais (UFMG) (RCF), Belo Horizonte, MG, Brasil; Faculdade de Medicina de Ribeirão Preto da Universidade de São Paulo

(FMRP-USP) (LJF, MCFF, ALDF, MCF); Faculdade de Ciências Farmacêuticas de Ribeirão Preto da Universidade de São Paulo (FCFRP-USP) (RCGA); Escola de Enfermagem de Ribeirão Preto da Universidade de São Paulo (EERP-USP) (AEP); Ribeirão Preto, SP, Brasil.

Recebido em 8/5/2008 Aceito em 20/10/2008

\author{
RESUMO
}

Objetivo: Descrever associações entre excesso de peso e obesidade abdominal com fatores de risco cardiovascular na população nipo-brasileira de Mombuca, Guatapará, SP. Métodos: Participaram do estudo 131 indivíduos com descendência japonesa $(69,2 \%$ da primeira e $30,8 \%$ da segunda geração), com idade $\geq 20$ anos, correspondendo a $66,8 \%$ da população residente dessa faixa etária. Os dados foram coletados por meio de questionários padronizados e foram realizados exames clínicos e laboratoriais. Resultados: A prevalência de sobrepeso foi de $29,6 \%$ entre os homens e de $25,6 \%$ entre as mulheres e a de obesidade foi de $46,3 \%$ entre os homens e de $39,0 \%$ entre as mulheres. Entre os homens a prevalência de obesidade abdominal foi de $55,6 \%$ e entre as mulheres de $20,8 \%$. 0 índice de massa corpórea foi associado independentemente com triglicérides, circunferência abdominal e idade; a pressão arterial sistólica com a circunferência abdominal. Conclusão: $O$ presente estudo enfatiza a necessidade de medidas de intervenção para a prevenção da obesidade na população nipo-brasileira de Mombuca. (Arq Bras Endocrinol Metab 2008; 52/9:1474-1481)

Descritores: Obesidade; Obesidade abdominal; Fatores de risco cardiovascuIar; Nipo-brasileiros

\section{ABSTRACT}

Obesity and its Relationship with Cardiovascular Risk Factors in a JapaneseBrazilian Population.

Objective: To describe associations of weight excess and abdominal obesity with cardiovascular risk factors in a Japanese-Brazilian population from Mombuca, Guatapará, SP. Methods: Cross-sectional study with 131 individuals of Japanese ancestry (69.2\% first and $30.8 \%$ second generation), aged 20 years or more, corresponding to $66.8 \%$ of the resident population from this age group. Data were collected through standardized questionnaires and laboratory and physical examinations were performed. Results: Prevalence of overweight and obesity was $29.6 \%$ and $46.3 \%$ in men and $25.6 \%$ and $39.0 \%$ in women, respectively. The prevalence of abdominal obesity was $55.6 \%$ in men and $20.8 \%$ in women. Body mass index was independently associated with triglycerides, waist circumference and age; systolic blood pressure with waist circumference. Conclusions: Data from the present survey highlight the necessity of interventions to prevent obesity in the Japanese-Brazilian population from Mombuca. (Arq Bras Endocrinol Metab 2008; 52/9:1474-1481)

Keywords: Obesity: Abdominal obesity; Cardiovascular risk factors; Japanese-Brazilians

\section{INTRODUÇÃO}

obesidade é considerada um problema de saúde pública em todo o mundo (1), e é destacada como fator de risco para uma série de doenças ou 
distúrbios, como a dislipidemia, a hipertensão arterial e o diabetes.

A avaliação da obesidade pode ser feita de várias maneiras, dependendo dos recursos disponíveis, finalidade e precisão. A antropometria é considerada o método mais útil para rastrear a obesidade, por ser de baixo custo, nãoinvasiva, universalmente aplicável, e com boa aceitação pela população (2). O índice de massa corpórea (IMC) é freqüentemente usado para avaliar a obesidade, tanto na clínica quanto em pesquisas; entretanto, a circunferência abdominal (CA) tem sido usada como medida de obesidade abdominal $(\mathrm{OA})$ e tem mostrado associação mais forte com fatores de risco para doenças cardiovasculares (DCV) do que o IMC (3-5).

Determinados grupos étnicos têm maior risco para a obesidade, fato que os predispõem a alguns tipos de doenças (6). A obesidade, especialmente a denominada do tipo central, cujo acúmulo de gordura se dá na região abdominal, pode indicar o início de uma série de alterações metabólicas adversas, que incluem a hiperinsulinemia, a tolerância à glicose diminuída, os níveis elevados de triglicérides (TG) e os níveis baixos de HDL-colesterol (HDL-c), o aumento de tendências trombogênicas e da síndrome metabólica (7).

Estudos com populações migrantes fornecem importantes contribuições para o conhecimento da etiopatogênese das doenças crônicas não transmissíveis (DCNT). Essas populações apresentam padrões distintos de morbimortalidade quando comparadas ao perfil dos residentes em seu local de origem, em conseqüência de mudanças nos seus hábitos e costumes, no seu processo de adaptação ao novo ambiente $(8,9)$.

As significativas mudanças socioculturais experimentadas por japoneses que migraram para o Ocidente, possivelmente estão associadas com a considerável elevação da mortalidade cardiovascular entre os imigrantes e seus descendentes nas Américas (9-11).

O Brasil alberga a maior comunidade de origem japonesa residente fora do Japão, porquanto $70 \%$ fixouse no estado de São Paulo (8). Inquérito realizado no ano 2000, na cidade de Bauru, SP, revelou alta prevalência de diabetes melito tipo 2 (DM2), ou seja, 34,9\%, e a freqüência de distúrbios lipídeos sangüíneos foi de $84 \%$, o que evidencia o impacto de alterações do estilo de vida, em particular do padrão alimentar, interagindo com possível suscetibilidade genética (8).

Na região de Ribeirão Preto, SP, mais precisamente no município de Guatapará, existe uma população de origem japonesa, que migrou mais recentemente (1962) e que, até os dias atuais, vive em uma comunidade semi-rural (distrito de Mombuca), e mantém grande parte de seus hábitos de vida tradicionais. A população adulta é composta, na sua maior parte, por issei (aqueles indivíduos que nasceram no Japão e migraram para o Brasil) e em menor proporção por nissei (filhos de issei). Uma população de origem japonesa, que ainda mantenha muito de seus hábitos de vida tradicionais, talvez apresente menor freqüência de excesso de peso e possivelmente menor risco para DCV.

O presente estudo se propôs a verificar a associação entre as medidas de obesidade avaliadas pelo IMC e $\mathrm{CA}$, com alguns fatores de risco para doenças cardiovasculares, na população nipo-brasileira de Mombuca, Guatapará, SP.

\section{MATERIAIS E MÉTODOS}

O estudo foi desenvolvido na comunidade nipo-brasileira de Mombuca, situada no município de Guatapará, no estado de São Paulo, no período de abril a dezembro de 2005 .

Para identificar a população residente na comunidade, foi realizado censo pela Associação Agro-Cultural e Esportiva de Guatapará, uma vez que a saída dos adultos é freqüente, em busca de trabalho no Japão ou em outras cidades brasileiras, e dos jovens, em virtude da conclusão dos estudos fora da comunidade. Neste levantamento foram identificados 196 indivíduos maiores de 20 anos.

Participaram do estudo 131 indivíduos com descendência japonesa ( $69,2 \%$ issei e $30,8 \%$ nissei), com idade $\geq$ 20 anos, o que representa $66,8 \%$ da população dessa faixa etária residente na comunidade, sendo $54(41,2 \%)$ do sexo masculino e $77(58,8 \%)$ do sexo feminino.

Os resultados apresentados são parte de um estudo transversal (diabetes e doenças associadas na população nipo-brasileira de Mombuca, Guatapará, SP), que foi aprovado pelo Comitê de Ética em Pesquisa do Centro de Saúde Escola da Faculdade de Medicina de Ribeirão Preto da Universidade de São Paulo; todos os participantes assinaram o termo de consentimento livre e esclarecido.

Foram realizadas reuniões, antes do início do estudo, com representantes da associação, e, posteriormente, com a comunidade de Mombuca em geral, para esclarecimentos sobre o inquérito e seus objetivos. 
Após a avaliação dos resultados dos exames clínicos e laboratoriais, os casos que necessitaram de acompanhamento médico foram encaminhados para a unidade de saúde conveniada ou Programa de Saúde da Família e para o Hospital das Clínicas da Faculdade de Medicina de Ribeirão Preto da Universidade de São Paulo (FMRP-USP), conforme a complexidade da situação.

Para a coleta dos dados, foram aplicados questionários que abordavam aspectos sociodemográficos, culturais, de saúde, atividade física e nutricionais, por entrevistadores treinados, a maioria alunos de graduação da Faculdade de Medicina de Ribeirão Preto (medicina e nutrição) e da Escola de Enfermagem de Ribeirão Preto da Universidade de São Paulo. A maioria das entrevistas foi acompanhada por intérpretes (indivíduos que viviam na comunidade e que dominavam a língua portuguesa), pelo fato de muitos entrevistados não serem fluentes com o português.

Quanto ao hábito de fumar, os participantes que relataram tabagismo atualmente foram classificados como fumantes; os que fumaram em alguma época da vida, mas que não fumam atualmente como ex-fumantes, e os que nunca fumaram como não-fumantes.

Os participantes classificaram sua atividade física, tanto no trabalho quanto no lazer, como leve, moderada, pesada ou muito pesada. Para a análise foram criadas três categorias: leve, moderada e intensa (atividade física pesada ou muito pesada).

O consumo de álcool foi categorizado como sim, quando os participantes relataram ingerir qualquer bebida alcoólica, mesmo que apenas socialmente, e como não, quando relataram não ingerir bebida alcoólica.

As avaliações antropométricas foram realizadas com os participantes descalços e com roupas leves. O peso foi avaliado em uma balança eletrônica (Filizola), com capacidade de $150 \mathrm{~kg}$ e precisão de $100 \mathrm{~g}$, com calibração prévia aos exames. A altura foi medida por antropômetro, com precisão de $0,5 \mathrm{~cm}$, colocado próximo à parede, em superfície plana, com o participante em posição ereta, pés unidos e calcanhares contra a haste de medida.

O IMC foi calculado mediante divisão do peso $(\mathrm{kg})$ pela altura $(\mathrm{m})$ ao quadrado.

Os participantes foram categorizados segundo os valores de IMC preconizados pelo Internacional Obesity Task Force (IOTF) (12) para a população asiática entre 23 e $24,9 \mathrm{~kg} / \mathrm{m}^{2}$ para sobrepeso e $\geq 25 \mathrm{~kg} / \mathrm{m}^{2}$ para obesidade.
Os valores da OA foram estimados pela medida da CA. O consenso apresentado pela International Diabetes Federation (IDF), em 2005 (13), mostra os valores de corte para a CA com base em gênero e etnia, e foram propostos para a população japonesa, os valores de $\geq 90$ $\mathrm{e} \geq 80 \mathrm{~cm}$, para homens e mulheres, respectivamente, utilizados neste estudo.

A CA foi medida passando a fita métrica à meia distância entre o rebordo inferior da última costela e a crista ilíaca; foram realizadas duas medidas para a circunferência, sendo utilizada a média dos dois valores. Para obtenção desta medida, empregou-se fita métrica não elástica, com precisão de $0,5 \mathrm{~cm}$.

Para verificar a pressão arterial, foi utilizado o aparelho automático da marca OMROM, modelo HEM 741 , com manguito para braços de 22 a $32 \mathrm{~cm}$, adequado para todos os participantes do estudo. A pressão arterial foi aferida três vezes, no braço direito a intervalos de 3 a 5 minutos, com o participante na posição sentada, pés no chão e pernas descruzadas, em repouso prévio de, pelo menos, 5 minutos. O valor final da pressão arterial foi a média das duas últimas medidas.

As amostras de sangue, coletadas em tubos a vácuo, com o participante em jejum de 10 a 14 horas, foram mantidas refrigeradas e transportadas para o Laboratório de Endocrinologia do Hospital das Clínicas de Ribeirão Preto, onde foram centrifugadas, aliquotadas e congeladas até a realização das dosagens.

As amostras de sangue para as dosagens da glicemia foram colhidas em tubos com fluoreto de sódio e dosadas por sistema enzimático (hexoquinase).

Para as dosagens de triglicérides (TG), de colesterol total (CT) e de HDL-colesterol (HDL-c), as amostras de sangue foram colhidas em tubo seco e foi utilizado o método automático espectrofotométrico no aparelho Integra 400, no Laboratório de Nutrição do Hospital das Clínicas de Ribeirão Preto. Os valores de LDL-colesterol (LDL-c) foram calculados segundo a fórmula de Friedwald, exceto quando os valores de triglicérides foram $\geq 400 \mathrm{mg} / \mathrm{dL}$.

A comparação de médias foi realizada pelo teste $\mathrm{t}$ de Student e o nível de significância adotado foi 0,05. A verificação da normalidade dos dados foi feita por meio da construção de gráficos (histogramas) de distribuição, bem como se aplicou o teste de KolmogorovSmirnov para normalidade. Utilizou-se a técnica de regressão linear para modelar a associação das variáveis dependentes, IMC e CA, com as independentes. 
As variáveis associadas à variável dependente na análise univariada, com valor de $\mathrm{p}<0,20$, e aquelas consideradas fatores de confusão, foram colocadas no modelo de regressão linear múltiplo para identificar as que mantinham associação de maneira independente, ao nível de $\mathrm{p}<0,05$. Para o modelo selecionado procedeu-se análise de resíduos, com o objetivo de testar as suposições do modelo de regressão linear e identificação de outliers, e este se mostrou adequado (14).

\section{RESULTADOS}

A Tabela 1 mostra as características gerais da população nipo-brasileira de Mombuca. A média de idade dessa população não apresentou diferença significativa entre os sexos, sendo de 55,1 anos para os homens e de 57,8 anos para as mulheres. Os valores médios de peso, altura, IMC e CA foram significativamente maiores entre os homens. As prevalências de sobrepeso e de obesidade foram, respectivamente, de $29,6 \%$ e de $46,3 \%$ entre os homens e de $15,6 \%$ e de $39 \%$ entre as mulheres, no entanto, essas diferenças não apresentaram significância estatística. Obesidade abdominal foi significativamente $(\mathrm{p}=0,01)$ mais freqüente entre os homens $(55,6 \%)$ do que entre as mulheres $(20,8 \%)$.

Os resultados da análise de regressão linear univariada mostraram associação estatisticamente significante $(\mathrm{p}<0,05)$ entre o IMC e as pressões arterial sistólica e

Tabela 1. Características da população nipo-brasileira estudada, Mombuca, Guatapará, SP, 2005. Dados expressos em média (desvio-padrão) e em número de indivíduos (percentual).

\begin{tabular}{lccc}
\hline \multicolumn{1}{|c}{ Variáveis } & $\begin{array}{c}\text { Homens }(\mathbf{n}=\mathbf{5 4}) \\
\text { Média }(\mathrm{dp})\end{array}$ & $\begin{array}{c}\text { Mulheres }(\mathbf{n}=\mathbf{7 7}) \\
\text { Média }(\mathrm{dp})\end{array}$ & p valor \\
\hline Idade (anos) & $55,1(15,9)$ & $57,8(15,9)$ & 0,32 \\
\hline Peso $(\mathrm{kg})$ & $70,6(4,5)$ & $55,2(10,2)$ & $<0,01$ \\
\hline Altura $(\mathrm{m})$ & $1,66(0,06)$ & $1,51(0,07)$ & $<0,01$ \\
\hline IMC $\left(\mathrm{kg} / \mathrm{m}^{2}\right)$ & $25,4(4,2)$ & $24,1(3,9)$ & 0,03 \\
\hline CA $(\mathrm{cm})$ & $88,6(10,1)$ & $83,1(10,7)$ & $<0,01$ \\
\hline & $\mathrm{n}(\%)$ & $\mathrm{n}(\%)$ & \\
\hline Sobrepeso & $16(29,6)$ & $12(15,6)$ & 0,08 \\
\hline Obesidade & $25(46,3)$ & $30(39,0)$ & 0,47 \\
\hline $\begin{array}{l}\text { Obesidade } \\
\text { abdominal }\end{array}$ & $30(55,6)$ & $16(20,8)$ & 0,01 \\
\hline
\end{tabular}

$\mathrm{IMC}$ = índice de massa corpórea; $\mathrm{CA}$ = circunferência abdominal. diastólica, os triglicérides e a CA. Houve associação inversa e estatisticamente significante $(\mathrm{p}<0,05)$ entre o IMC e o HDL-c e a idade, ou seja, à medida que os valores de IMC aumentavam, os níveis de HDL-c diminuiam e quanto maior a idade, menores os valores de IMC.

No modelo de regressão linear múltipla, apenas as variáveis triglicérides, CA e idade permaneceram associadas independentemente à variável resposta IMC (Tabela 2).

Quando se utilizou a CA como variável resposta, a análise de regressão linear univariada mostrou associação estatisticamente significativa $(\mathrm{p}<0,05)$ com glicemia de jejum, pressão arterial sistólica, triglicérides e IMC. Houve associação inversa e estatisticamente significante $(0,05)$ entre níveis de HDL-c e os valores de CA.

No modelo de regressão linear múltipla, apenas as variáveis pressão arterial sistólica e IMC permaneceram associadas independentemente à variável resposta CA (Tabela 3).

\section{DIscussão}

Os descendentes de japoneses que moram fora do Japão são mais suscetíveis ao DM2, dislipidemias e doenças isquêmicas do coração e cerebrovasculares. As mudanças no modo de vida, incluindo dietas ocidentais e diminuição de atividade física, juntamente com predisposição genética, estão envolvidas na deterioração do metabolismo da glicose, dos lipídios, no aumento de pressão arterial e na obesidade (9-11).

Um estudo de coorte realizado em 1994 com 8.823 japoneses residentes em várias cidades do Japão mostrou que o ganho de peso na idade adulta, mesmo com o indivíduo não apresentando quadro de obesidade, aumenta o risco para hipertensão e hipercolesterolemia, sendo igual ou até superior quando comparado com caucasianos (15).

O fenótipo da obesidade não é característica marcante dos migrantes japoneses residentes nas Américas. Entretanto, a ausência de IMC elevado não exclui a ocorrência de aumento na deposição visceral de gordura. Já se mostrou grande variabilidade na distribuição da gordura corporal em indivíduos com valores de IMC semelhantes $(9,11,16)$.

De acordo com o National Nutrition Survey in Japan (17), a prevalência de obesidade (IMC $\geq 25 \mathrm{~kg}$ / $\mathrm{m}^{2}$ ) nos homens e nas mulheres é de $30 \%$ e de $28 \%$, 
Tabela 2. Coeficientes de regressão, intervalos de confiança (IC) e p valor do modelo de regressão linear múltiplo. Variável dependente: IMC, na população nipo-brasileira de Mombuca, Guatapará, SP, 2005.

\begin{tabular}{|c|c|c|c|}
\hline Variáveis & Coeficiente de regressão & IC95\% & p valor \\
\hline Pressão arterial sistólica (mmHg) & 0,003 & $-0,022 ; 0,029$ & 0,784 \\
\hline Pressão arterial diastólica (mmHg) & 0,033 & $-0,013 ; 0,079$ & 0,160 \\
\hline $\mathrm{HDL}-\mathrm{c}(\mathrm{mg} / \mathrm{dl})$ & 0,004 & $-0,029 ; 0,037$ & 0,805 \\
\hline Triglicérides (mg/dl) & 0,006 & 0,$001 ; 0,010$ & 0,017 \\
\hline Circunferência abdominal (cm) & 0,265 & 0,$218 ; 0,313$ & 0,000 \\
\hline Idade (anos) & $-0,042$ & $-0,071 ;-0,013$ & 0,005 \\
\hline \multicolumn{4}{|l|}{ Consumo de álcool } \\
\hline Não & referência & & \\
\hline Sim & $-0,249$ & $-1,089 ; 0,590$ & 0,558 \\
\hline \multicolumn{4}{|l|}{ Tabagismo } \\
\hline Não fumante & referência & & \\
\hline Ex-fumante & 0,571 & $-0,613 ;-1,756$ & 0,342 \\
\hline Fumante & 1,022 & $-0,502 ; 2,547$ & 0,187 \\
\hline \multicolumn{4}{|l|}{ Sexo } \\
\hline Masculino & referência & & \\
\hline Feminino & 0,986 & 0,$012 ; 1,960$ & 0,050 \\
\hline \multicolumn{4}{|l|}{ Atividade física no lazer } \\
\hline Leve & referência & 0,$438 ; 2,181$ & 0,080 \\
\hline Moderada & 1,310 & $-1,455 ; 1,901$ & 0,793 \\
\hline Intensa & 0,222 & & \\
\hline
\end{tabular}

Tabela 3. Coeficientes de regressão, intervalos de confiança (IC) e p valor do modelo de regressão linear múltiplo. Variável dependente: CA, na população nipo-brasileira de Mombuca, Guatapará-SP, 2005.

\begin{tabular}{|c|c|c|c|}
\hline & Coeficiente de regressão & IC & p valor \\
\hline Glicemia de jejum (mg/dL) & 0,051 & $-0,017 ; 0,120$ & 0,140 \\
\hline Pressão arterial sistólica (mmHg) & 0,071 & 0,$019 ; 0,123$ & 0,008 \\
\hline Pressão arterial diastólica (mmHg) & 0,008 & $-0,067 ; 0,084$ & 0,826 \\
\hline $\mathrm{HDL}-\mathrm{c}(\mathrm{mg} / \mathrm{dL})$ & $-0,015$ & $-0,107 ; 0,076$ & 0,744 \\
\hline LDL-c (mg/dL) & $-0,023$ & $-0,053 ; 0,007$ & 0,129 \\
\hline Triglicérides (mg/dL) & 0,003 & $-0,013 ; 0,020$ & 0,684 \\
\hline $\mathrm{IMC}\left(\mathrm{kg} / \mathrm{m}^{2}\right)$ & 1,827 & 1,$489 ; 2,164$ & 0,000 \\
\hline Idade (anos) & 0,041 & 0,$035 ; 0,119$ & 0,286 \\
\hline \multicolumn{4}{|l|}{ Consumo de álcool } \\
\hline Não & referência & & \\
\hline Sim & 1,197 & $-1,053 ; 3,449$ & 0,294 \\
\hline \multicolumn{4}{|l|}{ Tabagismo } \\
\hline Não fumante & referência & & 0,775 \\
\hline Ex-fumante & 0,632 & $-3,742 ; 5,007$ & 0,713 \\
\hline Fumante & 0,777 & $-3,404 ; 4,958$ & \\
\hline \multicolumn{4}{|l|}{ Sexo } \\
\hline Masculino & referência & & \\
\hline Feminino & 2,161 & $-0,606 ; 4,928$ & 0,125 \\
\hline
\end{tabular}


respectivamente. Esses valores são inferiores aos evidenciados pelo presente estudo. A prevalência de sobrepeso foi de $29,6 \%$ entre os homens e de $25,6 \%$ entre as mulheres, e $46,3 \%$ dos homens e $39 \%$ das mulheres foram considerados obesos (IMC $\geq 25 \mathrm{~kg} / \mathrm{m}^{2}$ ). Em Bauru, a prevalência de excesso de peso (IMC $\geq 25 \mathrm{~kg}$ / $\mathrm{m}^{2}$ ) foi de $40,2 \%$ (18), valor semelhante ao encontrado no presente estudo, que, ao considerar o total dos indivíduos, evidenciou prevalência de $41,9 \%$ de obesidade.

O confronto da prevalência de obesidade na população de Mombuca com dados relativos à população geral brasileira apresenta limitações de ordem metodológica. No presente estudo, a utilização de ponto de corte mais baixo contribuiu para a elevada taxa de obesidade nos nipo-brasileiros, que ultrapassou a da população geral brasileira, e aproximou-se da dos Estados Unidos (19), onde estima-se que $40 \%$ a $50 \%$ dos adultos (20 a 74 anos) apresentem IMC acima de $25 \mathrm{~kg} / \mathrm{m}^{2}$.

A importância de determinados padrões de distribuição da gordura corporal é aventada desde o final dos anos 1950 (20). Atualmente sabe-se que é a localização abdominal da gordura (obesidade abdominal) que se mostra mais associada a distúrbios metabólicos, como as dislipidemias, a hipertensão arterial, a resistência à insulina, o DM2 e o risco cardiovascular (21). As medidas regionais de obesidade, entre as quais a $\mathrm{CA}$, são capazes de fornecer estimativa da gordura abdominal, que, por sua vez, está correlacionada à quantidade de tecido adiposo visceral (22).

A população de Mombuca apresentou valores médios de CA de $88,6 \mathrm{~cm}$ entre os homens e de $83,1 \mathrm{~cm}$ entre as mulheres, valores similares aos encontrados para o sexo masculino $(87,9 \mathrm{~cm})$ e feminino $(83,8 \mathrm{~cm})$ em nipo-brasileiros de Bauru (23), e inferiores aos relatados $(89,1 \mathrm{~cm})$ em nipo-americanos residentes em Seattle, Estados Unidos (24), para o sexo masculino. Os valores médios da população de Mombuca foram superiores aos dos japoneses residentes no Japão, tanto entre os homens $(84,1 \mathrm{~cm})$ quanto entre as mulheres $(73,2 \mathrm{~cm})(25)$.

A OA foi significantemente $(\mathrm{p}=0,01)$ mais freqüente entre os homens $(55,6 \%)$ do que entre as mulheres $(20,8 \%)$. Esses resultados foram inferiores aos encontrados pelo estudo de Bauru, que evidenciou prevalência de $\mathrm{OA}$ em $63,1 \%$ das mulheres e em $37,1 \%$ dos homens (18).

Na população nipo-brasileira de Bauru, a prevalência de $\mathrm{OA}(50,3 \%)$ foi superior à prevalência de obesi- dade $(40,2 \%)$ (18). Em Mombuca, foi evidenciado o contrário, a prevalência de obesidade $(41,9 \%)$ foi maior que a prevalência de $\mathrm{OA}(35,7 \%)$. Estudos sugerem que a combinação de análises de IMC e da distribuição da gordura aumenta o poder preditivo para distúrbios metabólicos e DCV (22).

Um estudo recente no Japão mostrou que as prevalências de $\mathrm{OA}$ entre os homens e as mulheres eram respectivamente, $54,8 \%$ e $5,8 \%$ (26). Os valores para os homens foram similares, porém, a prevalência de OA entre as mulheres de Mombuca foi bastante superior.

Em Mombuca, a prevalência de OA foi maior do que a de obesidade geral nos homens. Por outro lado, a prevalência de $\mathrm{OA}$ foi menor que a de obesidade geral nas mulheres. Muito se discute sobre os diferentes critérios para o diagnóstico de $\mathrm{OA}$ e obesidade geral em homens e mulheres. Essas diferenças podem influenciar a relação entre obesidade e fatores de risco cardiovascular em cada gênero. Estudos futuros são necessários para a melhor compreensão do significado da relação do IMC e da CA por gênero.

As análises de regressão linear univariada evidenciaram associações estatisticamente significantes entre as variáveis respostas IMC e CA e as variáveis independentes. No entanto, no modelo de regressão linear múltiplo, na presença de fatores de confusão, muitas associações perderam a significância estatística. Uma possível explicação é o tamanho da população, pois o número de participantes no estudo pode ter sido pequeno para evidenciar associações com significância estatística.

Uma das mais evidentes alterações que ocorre com o aumento da idade cronológica é a mudança nas dimensões corporais. Com o processo de envelhecimento, existem mudanças, principalmente na estatura, no peso e na composição corporal, com diminuição da massa muscular e da gordura corporal. Com essas mudanças, o IMC também se modifica, com o transcorrer dos anos (27). No presente estudo, a idade foi negativamente associada com os valores de IMC. Outra possível explicação seria o fato de os indivíduos de maior idade tenderem a manter hábitos de vida tradicionais, com menor ingestão de gordura e o processo de "ocidentalização" ter sido mais intenso na população mais jovem, o que gerou valores de IMC mais elevados.

A associação entre a obesidade e o aumento da pressão arterial tem sido mostrada tanto nos homens quanto nas mulheres, e em diversos grupos étnicos. Os 
estudos que buscaram comparar a associação do IMC e da CA com o aumento da pressão arterial chegaram a conclusões diversas. Ora evidenciaram superioridade do IMC, ora da circunferência abdominal $(28,29)$.

No presente estudo foi avaliada a associação entre valores de IMC e CA com os valores de pressões arteriais sistólica e diastólica. Os níveis de pressões arteriais não foram associados independentemente com os valores de IMC. Porém, os resultados indicam que a CA se associa de maneira importante com os níveis de pressão sistólica, mesmo após o controle de importantes variáveis de confusão, o que sugere que na população estudada, a CA seja indicadora mais sensível da associação entre obesidade e níveis pressóricos. Estudos realizados com populações ocidentais e asiáticas evidenciaram resultados semelhantes, em que a CA foi melhor preditivo de risco cardiovascular, incluindo níveis pressóricos e lipídicos (2,31).

A associação entre o excesso de peso e a dislipidemia foi verificada em diferentes estudos $(2,21)$. Nos indivíduos com sobrepeso e obesidade, freqüentemente, encontramos elevações leves ou moderadas dos níveis séricos de triglicérides e níveis séricos mais baixos de HDL-c; contudo, os níveis séricos de LDL-colesterol podem ou não estar aumentados (31).

Os resultados do presente estudo mostram apenas que o aumento dos valores de triglicérides está associado, independentemente, ao aumento do IMC. Apesar de a análise univariada ter evidenciado associação significativa e negativa entre HDL-c com o IMC e CA, quando se acrescentou no modelo as demais variáveis, essa associação perdeu a significância estatística.

Entre as limitações deste estudo, destaca-se o desenho transversal que não permite fazer inferências causais ou temporais acerca da associação entre variáveis dependentes e independentes. Estudos longitudinais futuros são necessários para avaliar a relação entre obesidade geral e obesidade abdominal e alguns fatores de risco para doenças cardiovasculares nesta população.

Embora a população de origem japonesa de Mombuca resulte de processo migratório mais recente, e até os dias de hoje mantenha parcela importante de seus hábitos tradicionais, ocorreu importante alteração em seu padrão alimentar, com maior consumo calórico diário, principalmente à custa de gordura, à semelhança do que foi observado em nipo-brasileiros de Bauru $(23,32,33)$.

O presente estudo enfatiza a necessidade de medidas de intervenção para a prevenção da obesidade na população nipo-brasileira de Mombuca. A utilização de parâmetros antropométricos simples, como ferramentas de triagem, pode ajudar na identificação de indivíduos com maior risco para doenças cardiovasculares e permitir intensificar medidas de prevenção.

Agradecimentos: À Fundação de Apoio ao Ensino, Pesquisa e Assistência do Hospital das Clínicas da Faculdade de Medicina de Ribeirão Preto da Universidade de São Paulo pelo apoio financeiro. À Associação Agro-Cultural e Esportiva de Guatapará pelo apoio prestado. À comunidade nipo-brasileira do Distrito de Mombuca pela participação neste estudo. Os autores declaram não haver conflitos de interesse científico neste artigo.

\section{REFERÊNCIAS}

1. World Health Organization. Diet, nutrition and the prevention of chronic diseases. Geneva: World Health Organization; 2003.

2. Ho SC, Chen YM, Woo JL, Leung SS, Lam TH, Janus ED. Association between simple anthropometric indices and cardiovascular risk factors. Int J Obes Relat Metab Disord. 2001;25:1689-97.

3. Dalton M, Cameron AJ, Zimmet PZ, Shaw JE, Jolley D, Dunstan DW, et al. Waist circumference, waist-hip ratio and body mass index and their correlation with cardiovascular disease risk factors in Australian adults. J Intern Med. 2003;254:555-63.

4. Zhu S, Wang Z, Heshka S, Heo M, Faith MS, Heymsfield SB. Waist circumference and obesity-associated risk factors among whites in the third National Health and Nutrition Examination Survey: clinical action thresholds. Am J Clin Nutr. 2002;76:743-9.

5. Foucan L, Hanley J, Deloumeaux J, Suissa S. Body mass index (BMI) and waist circumference (WC) as screening tools for cardiovascular risk factors in Guadeloupean women. J Clin Epidemiol. 2002;55(10):990-6.

6. Hill JO, Sydney S, Lewis CE, Tolan K, Scherzinger AL, Satmm ER. Racial differences in amounts of visceral adipose tissue in young adults: the CARDIA (Coronary Artery Risk in Young Adults) Study. Am J Clin Nutr. 1999;69(3):381-7.

7. Sociedade Brasileira de Cardiologia. Diretrizes para cardiologistas sobre excesso de peso e doença cardiovascular. Arq Bras Cardiol. 2002;78 Suppl 1:1-14.

8. Grupo de Estudos de Diabetes na Comunidade Nipo-Brasileira (JBDSG). Diabetes mellitus e doenças associadas em nipobrasileiros. São Paulo: Green Forest do Brasil Editora; 2004.

9. Fujimoto WY. The growing prevalence of non-insulin-dependent-diabetes in migrant Asian populations and its implications for Asia. Diabetes Res Clin Pract. 1992;15(2):167-83.

10. Gotlieb SL. Mortality among japanese migrants residing in the municipality of Sao Paulo, Brazil. Rev Saude Publica. 1990;24(6):453-67.

11. Franco LJ, Gimeno SGA, Ferreira SRG, lunes M, JapaneseBrazilian Diabetes Study Group (JBDSG). Incremento na mortalidade associada à presença de diabetes mellitus em nipo-brasileiros. Rev Saude Publica. 1998;32(2):118-24.

12. Weisell RC. Body mass index as an indicator of obesity. Asia Pac J Clin Nutr. 2002;11 Suppl 1: 681-4.

13. Alberti KGMM, Zimmet P, Shaw J. Metabolic syndrome-a new worldwide definition. A consensus statement from the International Diabetes Federation. Diabet Med. 2006;23(5):469-80. 
14. Neter J, Kutner MH, Nachtsheim CJ, Wasserman W. Applied linear statistical models. 4aㅡ. ed. Chicago: Irwin; 1996.

15. Ishikawa-Takata K, Ohta T, Moritaki K, Gotou T, Inoue S. Obesity, weight change and risks for hypertension, diabetes and hypercholesterolemia in Japanese men. Eur J Clin Nutr. 2002;56(7):601-7.

16. Hara H, Egusa G, Yamakido M, Kawate R. The high prevalence of diabetes mellitus and hyperinsulinemia among the Japanese-Americans living in Hawaii and Los Angeles. Diabetes Res Clin Pract. 1994;24 Suppl:S37-42.

17. Ministry of Health, Labor and Welfare, Japan. The National Nutrition Survey in Japan. Dai-ichi Shuppan, Tokyo; 2004.

18. Ferreira SRG, Lerario DDG, Gimeno SGA, Sanudo A, Franco LJ. Obesity and central adiposity in Japanese immigrants: role of the western dietary pattern. J Epidemiol. 2002;12(6):431-8.

19. Ardern $\mathrm{Cl}$, Katzmarzyk PT, Janssen I, Ross R. Discrimination of health risk by combined body mass index and waist circumference. Obes Res. 2003;11(1):135-42.

20. Vague J. The degree of masculine differentiation of obesities: a factor determining predisposition to diabetes, atherosclerosis, gout and uric calculus disease. Am J Clin Nutr. 1956;4:20-34.

21. McKeigue PM, Shah B, Marmot MG. Relation of central obesity and insulin resistance with high diabetes prevalence and cardiovascular risk in South Asians. Lancet. 1991;337:382-6.

22. Egger G. The case for using waist to hip ratio measurements in the routine medical checks. Med J Aust. 1992;156:280-5.

23. Taniguchi C, Gimeno SGA, Ferreira SRG, Japanese-Brazilian Diabetes Study Group (JBDSG). Características antropométricas de nipo-brasileiros. Rev Bras Epidemiologia. 2005;7(4):423-33.

24. Fujimoto WY, Bergstrom RW, Boyko EJ, Kinyoun JL, Leonetti DL, Newell-Morris LL, et al. Diabetes e diabetes risk factors in second and third-generation Japanese-Americans in Seattle. Diabetes Res Clin Pract. 1994;24(Suppl): S43-52.

25. Arai $\mathrm{H}$, Yamamoto A, Matsuzawa Y, Saito $Y$, Yamada N, Oikawa S, et al. Prevalence of metabolic syndrome in the general japanese population in 2000. J Atheroscler Thromb. 2006;13(4):202-8.

26. Hsieh SD, Muto T. Metabolic syndrome in Japanese men and women with special reference to the anthropometric criteria for the assessment of obesity: proposal to use the waist-toheight ratio. Prev Med. 2006;42:135-9.

27. Kannel WB, Wilson PW. Obesity, diabetes, and risk of cardiovascular disease in the elderly. Am J Geriatr Cardiol. 2002; 11(2):119-23.

28. Wildman RP, Gu D, Reynolds K, Duan X, Wu X, He J. Are waist circumference and body mass index independently associated with cardiovascular disease risk in Chinese adults? Am J Clin Nutr. 2005;82(6):1195-202.

29. Hayashi T, Boyko EJ, Leonetti DL, Marguerite MJ, Laura MN, Kahn SE, et al. Visceral adiposity and the risk of impaired glucose tolerance. Diabetes Care. 2003;26:650-5.

30. Ko GTC, Chan JCN, Woo J, et al. Simple anthropometric indexes and cardiovascular risk factors in Chinese. Int J Obes Relat Metab Disord. 1997;21:995-1001.

31. Katzel LI, Krauss RM, Goldberg AP. Relations of plasma TG and HDL-c concentrations to body composition and plasma insulin levels are altered in men with small LDL particles. Arterioscler Thromb Vasc Biol. 1994;14:1121-8.

32. Souza SMX. Análise do padrão alimentar dos nipo-brasileiros de Mombuca-Guatapará, SP, relacionando-o ao estado nutricional e alteração da homeostase glicêmica [dissertação de mestrado]. Ribeirão Preto, SP: Faculdade de Medicina de Ribeirão Preto, Universidade de São Paulo; 2006.

33. Freire RD, Cardoso MA, Shinzato AR, Ferreira SRG, JapaneseBrazilian Study Group (JBSG). Nutritional status of JapaneseBrazilian subjects: comparison across gender and generation. Br J Nutr. 2003;89(5):705-12.

\section{Endereço para correspondência:}

Roberta Carvalho de Figueiredo

Faculdade de Medicina da UFMG

Av. Professor Alfredo Balena, 190, $8^{\circ}$ andar, sala 814

30130-100 Belo Horizonte MG

E-mail: betafigueiredo@yahoo.com.br 